\title{
Attending to color with proper fixation
}

\author{
A. H. C. VAN DER HEIJDEN, A. G. KURVINK, L. DE LANGE, F. DE LEEUW, \\ and J. N. VAN DER GEEST \\ Leiden University, Leiden, The Netherlands
}

\begin{abstract}
In contemporary theorizing, there is a controversy about the role of spatial location in the selection of visual information; some theories postulate that position plays a unique role, whereas other theories hold that position is just one selection dimension that is not different from other dimensions, such as color and shape. In this context, a paradigm introduced by Tsal and Lavie (1988) promised to be of fundamental importance. With that paradigm, Tsal and Lavie found that, after reporting a first letter of a prespecified color, subjects preferred to switch their reporting to letters from array positions adjacent to that letter over continuing to report letters of the same color as that of the first letter. This switch from color to position provided firm evidence in favor of the "position-special" views as opposed to the "all-attributes-are-equal" views. In the present study, six experiments, employing Tsal and Lavie's paradigm and variations of that paradigm, are reported. Experiments 1, 2, 4, and 5 show that evidence for a switch from selection on the basis of color to selection on the basis of position is not obtained when subjects are forced to fixate the fixation point and possibly also not under normal contrast conditions without fixation controls. Experiment 3 shows that switching from color to position is difficult. Experiments 2, 5, and 6 show that evidence for a switch is obtained only under low-contrast conditions when subjects are not forced to fixate the fixation point. It is concluded that the Tsal and Lavie paradigm is an asymmetric paradigm. The results reported by Tsal and Lavie constituted a major threat for the "all-attributes-are-equal" theories and provided firm support for the "position-special" theories. The results reported in the present study are compatible with the all-attributes-are-equal theories, but, at the same time, do not constitute a major threat for the contemporary position-special theories.
\end{abstract}

In 1988, Tsal and Lavie could still write (p. 15) that the role of stimulus location in the selective processing of visual information had evolved into an implicit controversy; some researchers believed that spatial location played a unique role in the selection of information, whereas others claimed that it was just one selection dimension that was not different in principle from other stimulus dimensions, such as color or shape. Now, less than 10 years later, this implicit controversy has evolved into an explicit controversy (see, e.g., van der Heijden, 1993, for an overview). A brief look at current theorizing can make this clear. There are two groups of theories: the "position-not-special" ones and the "position-special" ones.

\section{Position Not Special}

In Broadbent's (1958) filter theory for auditory information processing, no special role was ascribed to position. The filter selected auditory information defined by any given physical attribute. ("Physical features identified as able to act as a basis for this selection included the intensity, pitch, and spatial localization of sounds"; Broadbent, 1958, p. 297.) Many later visual-informationprocessing theories can be regarded as generalizations of

The authors are grateful to Yehoshua Tsal, Claus Bundesen, Kyle Cave, and Jan Theeuwes for valuable suggestions and comments regarding this work. Correspondence should be directed to A. H. C. van der Heijden, Department of Experimental and Theoretical Psychology, P.O. Box 9555, 2300 RB Leiden, The Netherlands. this first theory (see, e.g., Broadbent, 1971; Kahneman, 1973; Neisser, 1967). So, also in these theories, position is merely one of the several attributes by which visual information can be selected.

Bundesen's (1990) position-not-special theory is currently the most sophisticated and detailed. Because all position-not-special theorizing culminates in it, this selective-attention theory deserves to be taken as the representative of the position-not-special views.

In the theory, a "front-end system" partitions the visual field into objects or elements against a background and calculates "measures of strength of sensory evidence concerning the nature of elements" in the visual field. In the analysis of experimental data, Bundesen assumes that these measures of strength are calculated in parallel across all objects in the visual field and across the perceptual categories of color, shape, and position. Object selection by color, form, or position is performed by manipulating "pertinence values"- -weights that indicate the current importance of attending to objects that belong to a certain perceptual category. For instance, for selecting the red elements in an array of red and black elements, the pertinence value for the category red is raised. An attentional mechanism translates these pertinence values into "attentional weights" by using the measures of sensory strength. As a result, all red elements receive relatively high attentional weights and all black elements receive relatively low ones. The relatively high weights cause the processing of all attributes of the red elements to be 
speeded up at the expense of the processing of the attributes of the black ones.

In this theory, the attentional weight is determined mainly by the strength of the sensory evidence that the element belongs to the desired subset of elements. It is therefore not the relevant dimension as such, but the discriminability within a relevant dimension that determines the ease and efficiency of object selection. So, in this theory, there is no principled distinction between selection of objects by position and selection of objects by color or shape.

\section{Position Special}

A great number of current visual-informationprocessing and selective-attention theories postulate a special role for position. In these theories, it is assumed that attentional selection is always ultimately accomplished via a representation that (also) portrays visual space. The "spotlight," "gradient," and "zoom lens" metaphors are examples of this theoretical position (see, e.g., Eriksen, 1990; Eriksen \& Hoffman, 1974; Eriksen \& Rohrbaugh, 1970; LaBerge \& Brown, 1989; Posner, Snyder, \& Davidson, 1980; see van der Heijden, 1992, for an overview).

Treisman's $(1988,1990)$ feature-integration theory is currently the most influential position-special theory. One of its major strengths is the recognition and use of the neurobiological discoveries of a multitude of separate visual areas that are specialized in coding different object properties (see, e.g., Felleman \& van Essen, 1991).

In Treisman's spotlight theory, there are two stages of processing. In a first, parallel, preattentive stage of processing, sensory features are registered independently by populations of feature detectors within specialized modules, for example, a color module, a form module, and a position module. The position module is described as a "master map of locations" that shows where all feature boundaries are located but not which features are located where (Treisman, 1988, p. 203). This module is placed at an earlier stage of analysis than the form module and the color module (see also Treisman \& Gormican, 1988). In a second, serial, attentive stage of processing, the sensory features are combined into whole objects. A serial spotlight of focused attention selects a particular position in the "master map of locations." A result of this attending at a selected position is that "the features currently present in the attended location are ... made available for further perceptual processing and can be combined in a representation of the attended object" (Treisman, 1990, p. 460).

In this theory, the spotlight of focused attention is directed at regions in "a master-map of locations that specifies where in the display things are, but not what they are" (Treisman \& Gormican, 1988, p. 17). So, attentional selection in vision is always ultimately attentional selection via position in visual space. Nevertheless, nonspatial attributes can indicate the position in the master map of locations where attention has to start. Attention "can sometimes be called to a particular location from a particular feature map.... The target feature activates its reciprocal link to the master map and allows attention to home in on its location" (Treisman, 1990, p. 460; see also Treisman, 1988, and Treisman \& Sato, 1990; for related theories, see, e.g., Cave \& Wolfe, 1990; van der Heijden, 1992, 1993, 1996; Wolfe, 1994, 1996; Wolfe, Cave, \& Franzel, 1989).

\section{A Paradigm}

The fact that nowadays position-special and positionnot-special theories coexist peacefully already indicates that relevant decisive information is hard to come by (see, e.g., Tsal \& Lavie, 1988, and van der Heijden, 1993, for overviews of the evidence). Either the evidence is simply irrelevant or the evidence is relevant but can be handled by both views when it is allowed to introduce additional assumptions (see van der Heijden, 1993). This state of affairs makes an ingenious paradigm, introduced by Tsal and Lavie (1988, Experiment 1), and the results they obtained with that paradigm, of fundamental importance for deciding between the two groups of theories. We first look at the paradigm and its importance and then at the results obtained.

Tsal and Lavie (1988, Experiment 1) presented their subjects with a circular array containing a mixture of three red, three green, and three brown letters. Letters of the same color were never adjacent. The subjects were instructed to report first a single letter of a given color, for example, a red one, and then any other letters they could identify. Tsal and Lavie considered the free report of "any other letters" as the important part of the experiment. This part was intended to reveal which attribute of the letter named first was attended to. In Tsal and Lavie's view, if attention is allocated to the location of this letter, adjacent letters should be reported more frequently than other letters in the display. If, however, attention is allocated to the color of the letter named first, letters of the same color should be reported more frequently than other letters in the display (see Tsal \& Lavie, 1988, p. 16).

Indeed, it seems that with this paradigm it would be possible to discriminate between position-not-special and position-special theories. A position-not-special theory, such as Bundesen's (1990) theory, assumes that the instruction "name first a red one" raises the pertinence value for the category red. Upon stimulus presentation, all red letters receive high attentional weights because there is strong sensory evidence that these letters are red. The other letters receive only low attentional weights. As a result, the processing of all attributes of the red elements is speeded up at the expense of the processing of the attributes of the differently colored letters, that is, the green and brown ones. Consequently, if position-not-special theories, such as Bundesen's, are correct about the operations of the internal information-processing system, then, in the free-report part, letters of the same color as the letter named first should be reported more frequently than other letters. 
A position-special theory, such as Treisman's (1988) theory, assumes that the instruction "name first a red one," together with activation of the feature "red" in the color module, guides the spotlight of attention to the position of one of the red letters in the "master map of locations." It is reasonable to assume that, after processing that letter, it is easiest to move the spotlight in the master map of locations to an adjacent position for processing letters for free report. Moreover, from several lines of research, it is well known that the hypothetical spotlight is always much too large (see, e.g., van der Heijden, 1992, chapter VI, for the evidence). So, it is highly likely that in the first fixation of the spotlight not only will the features of one of the red letters be made available for further perceptual processing, but so will the features of the neighboring, differently colored, letters. Consequently, if position-special theories, such as Treisman's, are correct about the inner workings of the visual information processing system, in the free-report portion of the task, letters adjacent to the one named first should be reported more frequently than other letters in the display (see, however, our General Discussion below).

Taken altogether, the Tsal and Lavie (1988) paradigm can be regarded as a tool for deciding between the positionspecial and position-not-special theories.

\section{The Results}

The results reported by Tsal and Lavie (1988, Experiment 1 ) are very clear and support the position-special theories. Among the additional free-report letters were significantly more letters from array positions adjacent to the letter named first than letters of the relevant color. So, even with a color instruction, position was dominant; after selecting the target letter on the basis of color, subjects went on to select the free-report letters mainly on the basis of position. Tsal and Lavie (1988) concluded that "the direction of attention to a relevant spatial location seems to be a general and mandatory process that takes place irrespective of the dimension according to which the stimulus was initially selected" (p. 19). Paradigm and data do indeed provide strong evidence in favor of positionspecial theories.

\section{A Problem}

Unfortunately, there is a major problem with Tsal and Lavie's (1988) color-position experiment, one that was noticed by the authors themselves: "A potential problem ... is that maintaining fixation at the center of the display may not have corresponded to the optimal strategy for performing the task.... The subjects may have shifted fixation to any array position prior to stimulus onset, since any random position was highly likely to include a letter of the relevant color in that or in an adjacent position. If subjects indeed shifted their gaze to one of the letter positions, then the superiority of location letters may reflect not the effect of attention, but the fact that these letters were simply closer to the focus than the remaining letters" (Tsal \& Lavie, 1988, p. 17). So, according to Tsal and Lavie, there is no need to invoke the operation of central selective attention; biased fixation and retinal acuity can produce a "pseudo spotlight" that can equally well account for the results obtained (see van der Heijden, 1992, pp. 55-59, for the importance of this factor).

\section{The Present Research}

Because of the potential importance of Tsal and Lavie's (1988, Experiment 1) paradigm and results for deciding between position-special and position-not-special theories, and because of the real possibility that the data obtained with this paradigm, which Tsal and Lavie report, are flawed because of inadequate fixation, we decided to repeat and extend their research. In the first four experiments, we investigated what happens with proper fixation. The last two experiments report what happens when there is no fixation control.

\section{EXPERIMENT 1}

The purpose of our first experiment was to replicate, with only one modification, Tsal and Lavie's (1988) experiment; to ensure correct fixation, we added another task to the letter-naming task. On each trial, at the point of fixation, a small digit appeared at the same time as the circular letter array. By being required to name this digit first, the subjects were forced to fixate the center of the display. A brief exposure time of the stimulus prevented them from making any directed eye movements during stimulus presentation.

From the literature, we know that there is not much reason to assume that this extra task severely influences the letter-naming task. Several investigators have demonstrated that letters and digits are processed rather independently when they are presented in a display simultaneously. One demonstration consists of the finding that lateral masking is strongly reduced when subjects have to find a target letter among digit distractors as opposed to the task of finding a target letter among letter distractors (see, e.g., Styles \& Allport, 1986). Another demonstration consists of the finding of the virtual absence of a display size effect for detecting a letter among digits, contrary to a large display size effect for detecting a letter among letters (see, e.g., Egeth, Jonides, \& Wall, 1972; see also Theeuwes, 1991). Variants of this method of presenting a digit pretask have been employed by LaBerge (1983) and LaBerge, Brown, Carter, Bash, and Hartley (1991), to vary the size of the attended area, and by Cohen and Rafal (1991), in a case study on feature integration in a patient with a parietal lobe lesion.

The subjects were instructed to report the digit first, then a single letter of a given color, and then any other letters they could identify. If the location of the first letter is at the basis of the attentional selection, letters adjacent to this first letter should be reported more frequently than other letters in the display. If the color of the first letter is attended to during selection, letters of the same 
color should be reported more frequently than other letters (however, see also our General Discussion below).

\section{Method}

Subjects. Eight students of the University of Leiden participated as subjects. All of them had normal or corrected-to-normal vision and none had a color deficiency. The subjects received a small amount of money for their services.

Stimuli. Each stimulus contained a circular array of letters formed by combining 132 different color sequences with nine uppercase letters. The letters were sampled randomly from the alphabet. The vowels were omitted, as well as the letter " $Q$ " because of its deviant form. Each array contained three red, three green, and three blue letters. The only two constraints were that letters of the same color never be adjacent and that letters never be repeated in a display. The spatial (and temporal) parameters were the same as in Tsal and Lavie's (1988) experiment. Each letter subtended $1^{\circ}$ of visual angle in height and $0.7^{\circ}$ in width of visual angle. The contour-tocontour interletter distance was $1.2^{\circ}$ of visual angle. The entire array subtended $6.6^{\circ}$ of visual angle in diameter. Simultaneously with the letters, a digit, randomly selected from the set $(2,3,4,5$, 6 ), appeared at the center of the display. The digit was enclosed in a small circle of $0.38^{\circ}$ of visual angle in diameter to make sure that it could not be identified without being properly fixated. Each digit appeared equally often.

Color specification. The letters were red (CIE $x y$-coordinates of $.62 / .35$, respectively, with a luminance of $27.2 \mathrm{~cd} / \mathrm{m}^{2}$ ), green $\left(.30 / .59,46.0 \mathrm{~cd} / \mathrm{m}^{2}\right)$, or blue $\left(.15 / .08,10.9 \mathrm{~cd} / \mathrm{m}^{2}\right)$. The background was black $\left(.29 /, 30, .1 \mathrm{~cd} / \mathrm{m}^{2}\right)$. The fixation point (a small dot) and the small circle with the digit were presented in white $\left(.29 / .31,126.2 \mathrm{~cd} / \mathrm{m}^{2}\right)$. All color characteristics were measured with a Photoresearch PR-703A spectrophotometer.

Apparatus. The experiment was conducted on a standard personal SX-486 computer. The computer controlled stimulus presentation. The subject, seated in front of a 14-in. VGA color monitor, controlled a mouse to activate the display. Viewing distance was $90 \mathrm{~cm}$. The experimenter entered the subject's responses into the computer.

Design. Each subject received 132 trials. These 132 different stimuli were presented in three blocks of 44 trials. In one block, the first letter to be named had to be red, in another block it had to be green, and in another, blue. The six possible presentation orders of block colors were randomized across subjects. The three experimental blocks were preceded by 22 practice trials, the results of which were not included in the analysis.

Procedure. First, the subjects were informed that the experiment was about reporting letters. They then read a screen text with information on the stimuli they were about to see and on the task they were to perform; if needed, additional procedural information was provided by the experimenter.

A trial started with a central fixation point (a small dot). When the fixation point was clearly seen, the subject could call the stimulus on the screen by pressing a mouse button. Each stimulus was presented for $100 \mathrm{msec}$. The subjects were instructed to first report the digit, then one letter in a given color, and then as many other letters as they could identify. It was emphasized that the purpose of the task was to report as many of the letters in the display as possible and that the letters might, but need not, be of the same color as the first letter. After the experimenter had entered the subject's response into the computer, the central fixation point reappeared and a new trial started.

\section{Results}

Before analyzing the data, we excluded the trials in which the digit was named incorrectly $(4.1 \%$ of the data) and the trials in which the first letter named was not in the required color or not in the display at all (13\% of the data). For the remaining trials, correct responses that reported letters adjacent to the first letter were classified as "location letters," letters that were of the same color as the first letter were classified as "color letters," and all other letters as "neutral letters." When subjects, after naming the first letter, reported additional letters that were not in the display, these letters were scored as "errors."

In an array of nine letters, there are two possible location letters, two possible color letters, and four possible neutral letters left after the first letter was named. We divided the number of letters in the neutral category by 2 , to make their number comparable to the number of letters in the other two categories. Also, we divided for each subject, in each block, his or her scores in the different categories by the number of correct trials for that subject in that block. This was done to make the scores between subjects and blocks comparable. The scores that were obtained by manipulating the data in this way-that is, the mean numbers of letters reported per trial for each factor combination - were used in the analysis of variance (ANOVA). Table 1 shows the means over subjects of the number of letters reported per trial in addition to the first letter, per letter category, and per color block, that is, red, green, and blue.

In the ANOVA, a highly significant effect was obtained for the factor of letter category $[F(2,14)=35.43, p<$ .0011 . The mean numbers of letters per category per trial were $0.23,1.05$, and 0.22 for location letters, color letters, and neutral letters, respectively. The factor of color block was not significant. The letter category $\times$ color block interaction approached significance $[F(4,28)=$ $2.62, .05<p<.10$; see Table 1].

\section{Discussion}

In Tsal and Lavie's (1988) Experiment 1, the subjects had to name one letter of a given color and then any other letters they could identify. Averaged over trials, the subjects named 2.66 letters ( 1 target and 1.66 other letters). In our Experiment 1, the subjects had to first name the digit, then one letter of a given color, and then any other letters they could identify. Averaged over the trials with a correct report of the digit, the subjects named 2.71 letters ( 1 target and 1.71 other letters). So, despite the addition of the digit-naming task, the subjects in our Experiment 1 performed as well as the subjects in Tsal and Lavie's Experiment 1. This finding is in accord with the

Table 1

Mean Number of Letters Reported per Trial in Addition to the First Letter, per Letter Category and per Color Block

\begin{tabular}{lccc}
\hline & Red & Green & Blue \\
\hline Location & 0.27 & 0.24 & 0.19 \\
Color & 0.88 & 1.03 & 1.25 \\
Neutral & $0.25\left(0.50^{*}\right)$ & $0.19\left(0.38^{*}\right)$ & $0.21\left(0.42^{*}\right)$
\end{tabular}

Note-The mean number of error letters per trial was 0.17 . ${ }^{*}$ Uncorrected mean. 
view that letters and digits are processed independently (see the introduction to this experiment).

The results show that in the present task subjects reported color letters much more often than other letters and that location letters were reported approximately equally often as neutral letters. While the letter category $\times$ color block interaction approached significance, the data in Table 1 show unambiguously that this pattern of letter reporting was obtained in all color blocks.

These findings strongly contradict the findings of Tsal and Lavie (1988), whose subjects named a significantly greater number of location letters than of other letters and showed a nonsignificant difference between color letters and neutral letters. Of course, it is highly likely that these diverging results are due to differences between our and Tsal and Lavie's experiments, and especially to the use of the central digit in the experiment here reported that forced subjects to fixate the center of the display. In Tsal and Lavie's experiment there was no such fixation control.

However, while "differences in fixation" is highly likely the important factor, there are two inadvertent differences between Tsal and Lavie's (1988) experiment and our experiment that are possibly (also) of importance and that deserve further investigation.

First, in our experiment, subjects had to name a digit plus letters. In Tsal and Lavie's experiment, subjects had to name letters only. It cannot be excluded that memory load or a related factor influenced the results obtained.

Second, we used a color monitor to present the stimuli. Tsal and Lavie used a tachistoscope for this purpose. It cannot be excluded that the discriminability of the stimuli was different in the two experiments, resulting in different effects or in the subjects' adopting different strategies (see also Wolfe et al., 1989, regarding the importance of this factor).

\section{EXPERIMENT 2}

Tsal and Lavie (1988) found a greater number of location letters than of other letters, while we found a greater number of color letters than of other letters. Before it can be concluded that differences in fixation, that is, "appropriate fixation" in our experiment and "shifted fixation" in Tsal and Lavie's experiment, are at the basis of this difference in results, two other possible explanations, based on differences in "memory load" and in "discriminability of the stimuli," have to be excluded. The investigation of these alternative explanations was the purpose of our second experiment.

The possibility that discriminability of the stimulus was responsible for the differences in results was investigated by varying this discriminability.

The possibility that the additional memory load or a related factor was responsible for the difference in results was investigated by changing the task used to ensure appropriate fixation from a digit-naming task into a go/nogo task, that is, by eliminating the additional memory load.

\section{Method}

Subjects. Eight students of the University of Leiden participated as subjects. All met the same requirements as the subjects of Experiment 1 . None of them had participated in the first experiment.

Stimuli. The stimuli were the same as those in Experiment 1 except that the small circle in the center of the display now contained either a plus (the "go" instruction) or a cross (the "no-go" instruction). The cross was set to appear randomly in $20 \%$ of the trials. Discriminability of the stimuli was varied by varying the letter-background contrast by using two light conditions (main light in the room: on and off, 40 and .4 lx, respectively) and two background conditions (screen background: white and black).

Color specification. For the letters, the same three colors (red, green, and blue) were used, as in Experiment 1. The background was either black $\left(.29 / .30, .1 \mathrm{~cd} / \mathrm{m}^{2}\right)$ or white $\left(.29 / .31,126.2 \mathrm{~cd} / \mathrm{m}^{2}\right)$. The fixation point and the small circle with the plus or cross symbol were presented in white when the background was black and in black when the background was white. All color characteristics were measured with the apparatus used in Experiment 1.

Apparatus. The apparatus was the same as that used in Experiment 1.

Design. The combination of the visibility factors of "light" (on and off) and "background" (white and black) resulted in the following four main letter-background contrast conditions: (1) Low contrast-colored letters on white background and main light on. (2) First in-between contrast-colored letters on white background and main light off. (3) Second in-between contrast--colored letters on black background and main light on. (4) High contrastcolored letters on black background and main light off. Each subject participated in all four main conditions. The presentation order of the four main conditions was varied randomly over subjects.

Per main condition, each subject received 132 trials. As in Experiment 1 , the 132 different stimuli were presented to them in three blocks of 44 trials that differed only in task color. The six possible presentation orders of block colors were randomized across the subjects.

The experiment was preceded by 45 general practice trials; in addition, each of the four main conditions was preceded by another 18 specific practice trials.

Procedure. First, the subjects were instructed, and then they practiced in nearly the same way as in Experiment 1 . The only differences concerned adaptation to the go/no-go instruction and to each of the four main conditions.

The experiment was run in two 1 -h sessions on 2 different days. On each day, a subject performed in two main conditions. So, the main procedure of the first experiment was now repeated four times for each subject, once for each of the letter-background contrast conditions.

\section{Results}

Before analyzing the data, we excluded the no-go trials (19.7\% of the data), the go trials without a response $(4.5 \%$ of the data), and the trials in which the letter named first was not in the required color or not in the display at all (9.5\% of the data). For the remaining trials, the responses in addition to the target letter were handled like the data used for analysis in Experiment 1 . The scores that were obtained by manipulating the data in this way were used in the ANOVA.

Table 2 shows the means over subjects of the number of letters reported per trial in addition to the first letter, per letter category and per main condition, that is, white/on, white/off, black/on, and black/off.

In the ANOVA, a significant main effect was obtained for the factor of letter category $[F(2,14)=7.65, p<.01]$. 
Table 2

Mean Number of Letters Reported per Trial in Addition to the First Letter, per Letter Category and per Main Condition

\begin{tabular}{lccccc}
\hline & \multicolumn{4}{c}{ Background } \\
\cline { 2 - 3 } \cline { 5 - 6 } & \multicolumn{2}{c}{ White } & \multicolumn{2}{c}{ Black } \\
\cline { 2 - 3 } \cline { 5 - 6 } & Light On & Light Off & & Light On & Light Off \\
\hline Location & 0.23 & 0.23 & & 0.23 & 0.26 \\
Color & 0.72 & 0.68 & 0.79 & 0.81 \\
Neutral & $0.30\left(0.60^{*}\right)$ & $0.23\left(0.46^{*}\right)$ & $0.30\left(0.60^{*}\right)$ & $0.30\left(0.60^{*}\right)$ \\
\hline
\end{tabular}

Note-The mean number of error letters per trial was 0.06 . ${ }^{*} \mathrm{Un}$ corrected mean.

The mean numbers of letters reported per trial in addition to the first letter were $0.24,0.75$, and 0.28 for location letters, color letters, and neutral letters, respectively.

None of the effects of the main visibility conditions were significant; the factor of light (on or off) was not significant, the factor of background (white or black) approached significance $(.05<p<.1)$, and so did the background $\times$ light interaction $(.05<p<.1)$. The mean numbers of letters reported per trial in addition to the first letter were $1.55,1.37,1.62$, and 1.67 in the four main contrast conditions white/on, white/off, black/on, and black/ off, respectively. There were no significant interaction effects of the factors of background and light with the factor of letter category.

Table 3 shows the mean numbers of letters reported per trial in addition to the first letter, per letter category, and per color block. The factor of color appeared to be significant $[F(2,14)=6.75, p<.01]$. The mean numbers of letters reported per trial in addition to the first letter were $1.51,1.56$, and 1.58 for the blocks red, green, and blue, respectively. The color $\times$ letter category interaction was highly significant $[F(4,28)=12.40, p<.001]$. This interaction is presented in Table 3 . There were no further significant effects.

\section{Discussion}

The results of the present experiment are very clear. In the four main contrast conditions and in all blocks, color letters were reported much more often than other letters and location letters and neutral letters were reported approximately equally often (see Tables 2 and 3 ). None of the further (significant) effects force us to qualify this pattern of results. So, in the present experiment, the same pattern of results was obtained as in our Experiment 1. (It is interesting to note that the average number of letters reported, 1.55, is about the same as that in Experiment $1,1.71$.)

Table 3

Mean Number of Letters Reported per Trial in Addition to the First Letter, per Letter Category and per Color Block

\begin{tabular}{lccc} 
& Red & Green & Blue \\
\hline Location & 0.25 & 0.26 & 0.20 \\
Color & 0.60 & 0.72 & 0.92 \\
Neutral & $0.33\left(0.66^{*}\right)$ & $0.29\left(0.58^{*}\right)$ & $0.23\left(0.46^{*}\right)$ \\
\hline
\end{tabular}

*Uncorrected mean.
The outcome of the present experiment strongly supports the point of view that the difference in results between our experiments and Tsal and Lavie's (1988) experiment is based on differences in fixation.

The absence of any reliable effect of the main visibility conditions indicates that discriminability of the stimulus is not an important determiner of task performance (see, however, Experiments 5 and 6). This finding therefore strongly suggests that it is highly unlikely that the difference in results obtained in our experiments and Tsal and Lavie's (1988) experiment is based on just this visibility factor.

The absence of any important differences between the outcome of our Experiment 1 with an additional memory load and the present experiment without a memory load indicates that memory load is also not an important determiner of task performance. This finding therefore indicates that it is highly unlikely that this factor is at the basis of the difference in results obtained in our experiments and Tsal and Lavie's (1988) experiment.

The only remaining important difference between our experiments and Tsal and Lavie's (1988) experiment seems to be the factor of fixation-"appropriate fixation" in our experiments and "shifted fixation" in Tsal and Lavie's experiment. It is therefore likely that the difference in selective performance is based on this factor.

\section{EXPERIMENT 3}

The instruction used by Tsal and Lavie (1988) and in our experiments was: "Name one letter in color $x$, and then as many other letters as you can identify. The other letters might, but need not, be of the same color as the first letter." With this instruction, at least two alternative strategies to perform the task are possible. One strategy consists of reporting as many letters of the same color as the first one as possible. Another strategy consists of reporting as many letters adjacent to the first letter as possible.

Because of this difference in possible strategies, we examined the effect of instructions. The digit-coloredletter stimuli in this experiment were the same as those used in Experiment 1. We presented each subject with two different, straightforward, instructions. In one session, the instruction was to name (after naming the digit) all three letters of the same color, that is, we explicitly told the subjects to select on the basis of color. In another session, the instruction was to name (after naming the digit) one single letter of a given color and then the two letters adjacent to this first one, that is, we explicitly instructed the subjects to select on the basis of location.

This experiment enabled us to assess, under conditions of proper fixation, with what type of instruction and corresponding strategy the task was easier to perform. The outcome can further strengthen our position that Tsal and Lavie's (1988) results are based on shifted fixation. If subjects appear to be better at selecting color letters than location letters, then the results of Tsal and Lavie's experiment can be explained by assuming that, with proper 
fixation, subjects adopted the difficult strategy to perform the task. That assumption is, however, theoretically unattractive. Given the short exposure time used and the time pressure under which the task had to be performed, it seems more likely that the subjects in Tsal and Lavie's experiment found another, easy strategy to perform the task, that is, shifted fixation to a part of the circular letter array before presentation of the stimulus.

\section{Method}

Subjects. Twelve students of the University of Leiden participated as subjects. All met the same requirements as the subjects in Experiment 1. None of them had participated in the previous experiments.

Stimuli, Color specification, and Apparatus. The stimuli, color specification, and apparatus were all the same as those used in Experiment 1

Design. There were two main conditions, a color-instruction condition and a location-instruction condition. In the colorinstruction condition, the subjects had to first name the digit and then all three letters of a given color. In the location-instruction condition, the subjects had to first name the digit, then one letter of the requested color, and finally the two letters adjacent to the letter named first.

In each main condition, each subject received 132 trials. The 132 different stimuli were presented in three blocks of 44 trials. In one block of the color-instruction condition, the subjects had to name the three red letters, in another block, the three green letters, and in the third block, the three blue letters. In one block of the location-instruction condition, the first letter they had to name was red, in another it was green, and in the third, blue. In both conditions, the six possible presentation orders of block colors were randomized across subjects.

Procedure. Each subject participated in one session with the color instruction and in one session with the location instruction. Each session was preceded by a practice block of 20 trials, the results of which were not included in the analysis. Both sessions were given on the same day, with a break of a few minutes in between. The order of the two sessions was balanced across subjects: 6 subjects participated first in the color-instruction condition and 6 subjects participated first in the location-instruction condition.

All other aspects of the procedure were the same as in Experiment 1 .

\section{Results}

Before analyzing the data, we excluded the trials in which the digit was named incorrectly ( $3.3 \%$ of the data), and the trials in which the first letter named was not in the required color or not in the display at all $(4.5 \%$ of the data). For the remaining trials, the responses in addition to the target letter were handled like the data used for analysis in Experiment 1. The scores that were obtained by manipulating the data in this way were used in the ANOVA. Table 4 shows the means over subjects of the number of letters reported per trial in addition to the first letter, per letter category, and per instruction.

The mean numbers of error letters per trial were 0.11 for the color instruction and 0.18 for the location instruction.

The ANOVA showed that the difference in performance due to the different instructions was highly significant $[F(1,11)=20.16, p<.001]$. The mean numbers of let-
Table 4

Mean Number of Letters Reported per Trial in Addition to the First Letter, per Letter Category and Per Instruction

\begin{tabular}{lcc}
\hline & \multicolumn{2}{c}{ Instruction } \\
\cline { 2 - 3 } & Color & Location \\
\hline Location & 0.05 & 0.87 \\
Color & 1.49 & 0.24 \\
Neutral & $0.14\left(0.28^{*}\right)$ & $0.16\left(0.32^{*}\right)$ \\
\hline
\end{tabular}

*Uncorrected mean.

ters per trial were 1.82 in the color-instruction condition and 1.43 in the location-instruction condition. The factor of letter category was also highly significant $[F(2,22)=$ $84.71, p<.001]$. Averaged over instructions, the subjects named 0.46 location letters, 0.87 color letters, and 0.31 neutral letters. The interaction between the factors of instruction and letter category was also highly significant $[F(2,22)=150.46, p<.001]$. This interaction is presented in Table 4.

\section{Discussion}

In the present experiment, we used two very clear, unambiguous instructions. In one condition, we asked subjects to name one letter of a given color and then the other two letters of the same color. In the other condition, we asked them to name one letter of a given color and then the two letters that were adjacent to the first letter. The results show that subjects are able to perform both tasks. The highly significant interaction between the factors of instruction and letter category indicates that, with the color instruction, subjects named more color letters than location letters ( 1.49 and 0.05 , respectively), and that with the location instruction, they named more location letters than color letters $(0.87$ and 0.24 , respectively).

The results, however, also show that subjects cannot perform the tasks equally well. When the task is to name the two other letters of the same color after naming the first one, subjects report, on the average, 1.82 letters, including 1.49 color letters. When the task is to name the two adjacent letters after naming the first one, subjects report on the average 1.43 letters, including 0.87 location letters. These data and the ANOVA make it clear that the location-instruction task is far more difficult to perform than the color-instruction task.

In our Experiments 1 and 2, we found mainly selection of color letters. Given the outcome of the present experiment, this result is easy to understand. With proper fixation, it is easier to proceed with reporting color letters after the first letter than it is with reporting location letters. So the subjects in our experiments simply performed the task by following the easiest strategy.

In Tsal and Lavie's (1988) experiment, the subjects named more location letters than color letters. This outcome, too, is easy to understand. The subjects can perform the task with either appropriate fixation or shifted fixation. The results of the present experiment show that, with "appropriate fixation," naming location letters is more difficult than naming color letters. In general, sub- 
jects seldom choose to perform a task using a difficult strategy when an easier strategy is available; our Experiments 1 and 2 are demonstrations of this point. It is therefore unlikely that the subjects in Tsal and Lavie's experiment performed under conditions of appropriate fixation. So the shifted fixation hypothesis offers the most parsimonious explanation of Tsal and Lavie's location-selection results (see, however, our General Discussion below).

\section{EXPERIMENT 4}

Experiments 1 and 2 showed that, with proper fixation in the center of the array, subjects do not switch from selection by color to selection by position, as reported by Tsal and Lavie (1988, Experiment 1). After having selected the first target on the basis of color, subjects maintain their selection strategy and also select the letters for additional free report mainly on the basis of color. Experiment 3 made clear why subjects prefered to perform the task this way. This experiment showed that better performance is achieved when selection on the basis of color is maintained than when a switch is made from selection on the basis of color to selection on the basis of position. So, in Experiments 1 and 2, the subjects simply performed the task in the easiest and most adequate way.

The results of our first three experiments add to the already available evidence that subjects are very well capable of selecting subsets of visual information on the basis of color (for further evidence, see, e.g., Bundesen, Pedersen, \& Larsen, 1984; Bundesen, Shibuya, \& Larsen, 1985; Clark, 1969; Francolini \& Egeth, 1980; Fryklund, 1975; Humphreys, 1981; Kaptein, Theeuwes, \& van der Heijden, 1995; Nissen, 1985; Snyder, 1972; von Wright, 1968, 1970, 1972). Nevertheless, it is worthwhile to note that our evidence for efficient selection on the basis of color was obtained in a particular situation. Just as in Tsal and Lavie's experiment, the color of the first letter to be named was constant in a whole block of trials. This "consistent cuing" might have introduced a consistent, strong "expectation" (see van der Heijden, 1992, chapter II). Therefore, it cannot be excluded that not selective attention, but a consistent expectation, is the basis for the evidence of efficient selection by color that we obtained.

To establish the properties of selection by color further, we performed an experiment with proper fixation in which the "consistent expectation" was eliminated so that selective attention would need to make the selection. We investigated whether with "varied cuing" the same strong evidence for selection made on the basis of color would be obtained. Before each trial, we presented a small, colored plus sign at the fixation point. The color of the plus sign was red, green, or blue. The color indicated the color of the first letter the subjects were to name. The sequence of colors was random. The size of the plus sign was such that the color could be discerned only when it was fixated.

\section{Method}

Subjects. Eight students of the University of Leiden participated as subjects. All met the same requirements as the subjects in Experiment 1. None of them had participated in the previous experiments.

Stimuli. The stimuli differed from the stimuli in Experiment 1 only in that the small circle in the center of the display now contained a small plus sign ( + ) that was red, green, or blue. The color of the plus sign could not be identified without proper fixation.

Color specification. For the letters, the background, the fixation point, and the small circle, the colors were the same as those used in Experiment 1. For the plus sign in the small circle, the colors red, green, and blue were the same as those used for the letters.

Apparatus. The apparatus was the same as that used in Experiment 1 .

Design. Each subject received 132 trials. These 132 different stimuli -44 with a red, 44 with a green, and 44 with a blue plus sign - were presented to them in a random order.

Procedure. After first being instructed, the subjects practiced in almost the same way as in Experiment 1 . The differences concerned only adaptation to the color instruction, that is, the interpretation and use of the colored plus sign. The instruction was: "Name first a letter with the same color as the plus sign and then as many other letters as you can identify. The other letters may, but need not be, of the same color as the first letter."

\section{Results}

Before analyzing the data, we excluded the trials in which the first letter named was not in the required color or not in the display at all ( $16.1 \%$ of the data). For the remaining trials, the responses in addition to the target letter were handled like the data used for analysis in Experiment 1 . The scores that were obtained by manipulating the data in this way were used in the ANOVA.

Table 5 shows the corresponding means over subjects of the number of letters reported per trial in addition to the first letter, per letter category, and per color.

The ANOVA showed a highly significant effect for the factor of letter category $[F(2,14)=70.57, p<.001]$. The mean numbers of letters per trial were $0.16,0.45$, and 0.16 for location letters, color letters, and neutral letters, respectively. There were no further significant effects.

\section{Discussion}

The results of the present experiment are similar to those of Experiments 1 and 2. The effect of letter category is highly significant; just as in Experiments 1 and 2, color letters were reported much more often than other letters, and location letters and neutral letters were reported approximately equally often. This result indicates that subjects are able and prefer to select letters on the basis of

Table 5

Mean Number of Letters Reported per Trial in Addition to the First Letter, per Letter Category and per Color

\begin{tabular}{lccc}
\hline & Red & Green & Blue \\
\hline Location & 0.18 & 0.14 & 0.16 \\
Color & 0.48 & 0.43 & 0.45 \\
Neutral & $0.17\left(0.35^{*}\right)$ & $0.15\left(0.30^{*}\right)$ & $0.15\left(0.29^{*}\right)$ \\
\hline
\end{tabular}

Note-The mean number of error letters per trial was 0.34 . ${ }^{*}$ Uncorrected mean. 
color, even if they are informed of the color of the letter to be selected first on a trial-by-trial basis and almost simultaneously with the letter stimulus. This result suggests that the findings obtained in Experiments 1 and 2 need not be attributed to the "consistent cuing" procedure that was used or to the consistent strong "expectation" that was induced. It is therefore reasonable to conclude that a flexible operation of selective attention is involved in the efficient selection by color that we obtained in our experiments.

In the present experiment, the letter-category effect $(0.16,0.45$, and 0.16 for location letters, color letters, and neutral letters, respectively), while highly consistent, is somewhat smaller than the letter-category effects obtained in Experiment $1(0.23,1.05$, and 0.21) and Experiment $2(0.24,0.75$, and 0.28$)$. Also, the number of letters reported correctly $(.93)$ is somewhat lower than those found in Experiment 1 (1.70) and Experiment 2 (1.57), while the number of error letters $(0.34)$ is somewhat higher than those found in Experiment $1(0.17)$ and Experiment 2 $(0.06)$. These results suggest that the "varied cuing" version employed here was appreciably more difficult than the "consistent cuing" versions employed earlier. Further research is needed to assess precisely what factor is responsible for this difference in difficulty.

\section{EXPERIMENT 5}

Experiments 1, 2, and 4 convincingly show that, in the Tsal and Lavie (1988) paradigm, under conditions of proper eye fixation, subjects, after they have selected a first target on the basis of color, prefer to continue with selection on the basis of color for additional free report. Experiment 3 indicated why this was so. With proper fixation, naming color letters is easier than naming location letters.

As stated in the general introduction to this paper, Tsal and Lavie (1988, p. 17) had already surmised that in their experiment, instead of looking at the fixation cross, subjects may have shifted fixation to any array position prior to stimulus onset, since any random position was highly likely to include a letter of the relevant color in that or an adjacent position. But, if subjects indeed prefer to shift their fixation point, the conclusion has to be that naming color letters is not really easy.

This outcome - that naming color letters is easier than naming location letters but nevertheless is not really easymakes the question of under what conditions Tsal and Lavie's (1988) results-more location letters than color letters-can be obtained really intriguing. This question is investigated in this experiment and in the following one.

To investigate whether subjects prefer shifting their fixation point over naming color letters with proper fixation, an experiment without any fixation controls - digits, pluses, or colors-was performed. Just as in Tsal and Lavie's (1988) experiment, only a central fixation cross preceded the stimulus and subjects were simply instructed to fixate that cross. If, under these conditions,
Tsal and Lavie's results were to be obtained, it would be reasonable to conclude that subjects prefer not to follow the instructions and, instead, to shift their fixation to one of the array positions. If, however, the results were the same as those obtained in our earlier experiments, more is involved, and a further search for the exact conditions under which fixation shifts are provoked would be needed.

\section{Method}

Subjects. Eight students of the University of Leiden participated as subjects. All met the same requirements as had the subjects of Experiment 1. None of them had participated in the previous experiments.

Stimuli. The stimuli differed from those in Experiment 1 only in that there was no central circle with a digit.

Color specification, Apparatus, and Design. For all the elements of the stimuli, the colors were the same as those used in Experiment 1 . The apparatus and design were the same as in Experiment 1 .

Procedure. The procedure was almost the same as in Experiment 1 . The only difference consisted in the adaptation of the instruction and the task to the alteration in the stimuli.

\section{Results}

Before analyzing the data, we excluded the trials in which the first letter named was not in the required color or not in the display at all (5.0\% of the data). For the remaining trials, the correct responses in addition to the target letter were handled like the data used for analysis in Experiment 1 . The scores that were obtained by manipulating the data in this way were used in the ANOVA.

Table 6 shows the corresponding means over subjects of the number of letters reported per trial in addition to the first letter, per letter category, and per color block.

In the ANOVA a significant effect was obtained for the factor of letter category $[F(2,14)=8.90, p<.01]$. The mean numbers of letters per trial in addition to the first letter were $0.48,1.19$, and 0.24 for location letters, color letters, and neutral letters, respectively. The subjects named more color letters than either location $[t(7)=$ $2.249, p<.05]$ or neutral letters $[t(7)=4.222, p<.01]$ and more location letters than neutral letters $[t(7)=$ $2.036, p<.05]$.

The factor of color block was significant $[F(2,14)=$ $4.71, p<.05]$. The mean numbers of letters reported per trial in addition to the first letter were 2.16,2.05, and 2.25 in blocks red, green, and blue, respectively. The interaction between color block and letter category was not significant.

Table 6

Mean Number of Letters Reported in Addition to the First Letter, per Letter Category and Per Color Block

\begin{tabular}{lccc}
\hline & Red & Green & Blue \\
\hline Location & 0.44 & 0.48 & 0.52 \\
Color & 1.22 & 1.07 & 1.29 \\
Neutral & $0.25\left(0.50^{*}\right)$ & $0.25\left(0.50^{*}\right)$ & $0.22\left(0.44^{*}\right)$ \\
\hline
\end{tabular}

Note-The mean number of error letters per trial was 0.13 . ${ }^{*} \mathrm{Un}-$ corrected mean. 
Table 7

Mean Number of Letters Reported per Trial in Addition to the First Letter, per Subject and per Letter Category

\begin{tabular}{cccc}
\hline & \multicolumn{3}{c}{ Letter Category } \\
\cline { 2 - 4 } Subject & Color & Location & Neutral \\
\hline 1 & 1.67 & .29 & .13 \\
2 & .89 & .29 & .37 \\
3 & 1.41 & .58 & .39 \\
4 & 1.75 & .12 & .14 \\
5 & 1.23 & .49 & .18 \\
6 & 1.70 & .03 & .08 \\
7 & .42 & 1.19 & .31 \\
8 & .45 & .83 & .33 \\
\hline
\end{tabular}

\section{Discussion}

In Experiments 1, 2, and 4, with fixation controls, subjects reported color letters more often than other letters and location letters approximately equally as often as neutral letters. In the present experiment, without fixation controls, the outcome was somewhat different. Subjects still reported more color letters than other letters, but location letters were now reported more frequently than neutral letters. Table 7 shows what is at the basis of this difference in results. Subjects 1-6 show a pattern of performance similar to that of the subjects in Experiments 1,2, and 4. Subjects 7 and 8, however, show a different pattern of results; they show the pattern of performance reported by Tsal and Lavie (1988).

The finding that 6 of the 8 subjects performed as in Experiments 1, 2, and 4 is very fortunate for two different reasons. First, it demonstrates that it was not the extra "fixation" task that made the results we obtained in Experiments 1, 2, and 4 differ from Tsal and Lavie's (1988) results. Second, this finding strongly suggests that most subjects experienced color selection with proper fixation as not so difficult as to be better replaced by random selection with shifted fixation.

The results of this experiment show that the absence of fixation controls only is not a sufficient condition for provoking fixation shifts to an array position prior to stimulus exposure, and, consequently, also not for producing the pattern of results reported by Tsal and Lavie (1988, Experiment 1). In other words, the results show that, besides the absence of fixation controls, still another factor must be involved.

\section{EXPERIMENT 6}

It cannot be excluded that subjects tend to follow the instruction to fixate the fixation point whenever possible and that they start refusing to obey this instruction only when the experimental conditions provoke them to do so. In Experiment 5, rather optimal visibility conditions were used. It is possible that the visibility conditions were appreciably worse in Tsal and Lavie's tachistoscope experiment (see also our introduction to Experiment 2). It is even possible that, with central fixation, the letters were not clearly perceived. If so, it is possibly "nonoptimal visibility conditions" which, in combination with "ab- sence of adequate fixation controls," provokes fixation shifts to an array position before stimulus presentation.

To test the hypothesis that fixation shifts are provoked under nonoptimal visibility conditions and in the absence of fixation controls, we decided to replicate Experiment 5 under less than optimal visibility conditions. To this end, we used one of the in-between contrast conditions from Experiment 2-a low-contrast condition with a white background and the main room light off. In this condition, the stimuli closely resemble the stimuli used by Tsal and Lavie (1988). ${ }^{1}$ If Tsal and Lavie's results were obtained under this condition, it is reasonable to conclude that subjects did not obey the instruction and shifted fixation to one of the array positions.

\section{Method}

Subjects. Eight students of the University of Leiden participated as subjects. All met the same requirements as did the subjects of Experiment 1. None of them had participated in the previous experiments.

Stimuli. The stimuli were identical to those in Experiment 5. They were displayed as in the first in-between contrast condition in Experiment 2-colored letters on a white background and main light off.

Color specification. The colors were the same as those used in the first in-between contrast condition of Experiment 2.

Apparatus and Design. The apparatus and design were the same as those used in Experiment 1

Procedure. The procedure was the same as that used in Experiment 5.

\section{Results}

Before analyzing the data, we excluded the trials in which the first letter named was not in the required color or not in the display at all $(3.7 \%$ of the data). For the remaining trials, the responses in addition to the target letter were handled like the data used for analysis in Experiment 1. The scores that were obtained by manipulating the data in this way were the data we used in the ANOVA.

Table 8 shows the corresponding means over subjects of the number of letters reported per trial in addition to the first letter, per letter category, and per color block.

In the ANOVA, a significant effect was obtained for the factor of letter category $[F(2,14)=8.69, p<.05]$. The mean numbers of letters per category were $0.79,0.55$, and 0.27 for location letters, color letters, and neutral letters, respectively. The subjects reported more location letters than neutral letters $[t(7)=4.877, p<.001]$ and more color letters than neutral letters $[t(7)=3.598, p<.01]$. The difference between location letters and color letters

Table 8

Mean Number of Letters Reported in Addition to the First Letter, per Letter Category and per Color Block

\begin{tabular}{lccc}
\hline & Red & Green & Blue \\
\hline Location & 0.77 & 0.91 & 0.70 \\
Color & 0.51 & 0.40 & 0.73 \\
Neutral & $0.29\left(0.58^{*}\right)$ & $0.30\left(0.60^{*}\right)$ & $0.23\left(0.46^{*}\right)$
\end{tabular}

Note-The mean number of error letters per trial was 0.06 . ${ }^{*}$ Uncorrected mean. 
Table 9

Mean Number of Letters Reported per Trial in Addition to the First Letter, per Subject and per Letter Category

\begin{tabular}{cccc}
\hline & \multicolumn{3}{c}{ Letter Category } \\
\cline { 2 - 4 } Subject & Color & Location & Neutral \\
\hline 1 & .48 & .92 & .23 \\
2 & .59 & .88 & .42 \\
3 & .29 & 1.37 & .27 \\
4 & .34 & .88 & .21 \\
5 & .57 & .84 & .36 \\
6 & .45 & .45 & .19 \\
7 & .89 & .71 & .45 \\
8 & .79 & .28 & .07 \\
\hline
\end{tabular}

was not significant $[t(7)=1.413]$. There were no further significant effects.

\section{Discussion}

In Experiment 5, subjects reported more color letters than location letters. In the present experiment, with less favorable exposure conditions, this difference disappeared. There is even some indication, albeit not significant, of the opposite result. Table 9 shows what is at the basis of the results obtained. Only Subject 8 still clearly shows the pattern of results that we found in Experiments 1,2, and 4 and, for the majority of subjects, in Experiment 5 . Subjects $1-5$ show a pattern of performance similar to that reported by Tsal and Lavie (1988), and Subjects 6 and 7 show an intermediate pattern of results.

While the present experiment is not a perfect replication of Tsal and Lavie's (1988) experiment, it provides us with sufficient information for explaining the results they obtained. From Experiment 2, we already know that "less than optimal exposure conditions" alone is not a sufficient condition for producing the pattern of results they report. From Experiment 5, we already know that the "absence of fixation controls" is also not a sufficient condition for producing that result. The present experiment shows that, with the combination of the "less than optimal exposure" and "absence of fixation controls" conditions, a pattern of performance closely similar to that reported by Tsal and Lavie is obtained. It is therefore reasonable to conclude that, in the absence of fixation controls, less than optimal exposure conditions provoke fixation shifts and that shifted fixations are at the basis of Tsal and Lavie's results (see, however, our General Discussion below).

\section{GENERAL DISCUSSION}

As stated in our general introduction, there is nowadays an explicit controversy about the role of stimulus location in the selective processing of visual information; some researchers believe that spatial location plays a unique role in the selection of visual information (see, e.g., Treisman, 1988; Treisman \& Gormican, 1988; van der Heijden, 1992, 1993), whereas others claim that position is just one selection dimension that is not differ- ent from such other dimensions as color and shape (see, e.g., Bundesen, 1990).

In this theoretical context, the ingenious paradigm introduced by Tsal and Lavie (1988) promised to be of fundamental importance. The paradigm and the results obtained with that paradigm seemed to provide firm evidence in favor of the position-special views and against the position-not-special or all-attributes-are-equal views. With their paradigm, Tsal and Lavie found that, after report of a first letter of a prespecified color, subjects preferred to continue free report with letters from array positions adjacent to that letter and not with letters of the same color as that first letter.

For the position-special views, such a switch from color to position is not really unexpected. According to these views, selection in vision is always ultimately selection on the basis of position. These theories can therefore assume that, after a first selection on the basis of color and position, subjects simply neglect the color dimension as a selection criterion and continue with selection on the basis of position only. So, according to these theories, no fundamental change in selection strategy occurs.

For the all-attributes-are-equal theories, such a switch from color to position is really unexpected. According to these theories, only color is involved in selection on the basis of color. The location dimension is irrelevant and, therefore, not involved in the selection of the first letter in Tsal and Lavie's (1988) task. So these theories need to explain why subjects make a fundamental change in their selection strategy, that is, that they prefer to switch from selection only on the basis of color to selection only on the basis of position. It is very difficult to see how such an explanation can be framed without assuming, in one way or another, that, in selection in vision, position plays a special role (see van der Heijden, 1993, and also Bundesen, 1991).

The results of the experiments reported in this study show, however, that the situation for the all-attributes-areequal theories is certainly not as desperate as Tsal and Lavie's (1988) research suggested. In this respect, two outcomes of our experiments are worth emphasizing.

First, from our Experiments 2, 5, and 6, it can be concluded that Tsal and Lavie's (1988) evidence for a switch from selection on the basis of color to selection on the basis of position is obtained only under less favorable exposure conditions when subjects are not forced to fixate the fixation point. Of course, these are exactly the conditions that invite fixation shifts to an array position prior to stimulus exposure, and such fixation shifts can explain the pattern of results that Tsal and Lavie obtained (see our general introduction and also Tsal \& Lavie, 1988 , p. 17). But, if subjects really shifted fixation in Tsal and Lavie's experiment and in our Experiment 6, the results of these experiments tell us nothing of importance about central selection in vision.

Second, from our Experiments 1,2, 4, and 5, it appears that Tsal and Lavie's (1988) evidence for a switch from selection on the basis of color to selection on the basis of 
position is not obtained when subjects are forced to fixate the fixation point (Experiments 1, 2, and 4) and, for most subjects, even not under adequate exposure conditions without fixation controls (Experiment 5). Because directed eye movements were excluded in Experiments 1,2 , and 4 , these experiments undoubtedly measure central selection in vision. It can therefore be concluded that, under appropriate conditions in the Tsal and Lavie paradigm, subjects prefer not to switch selection strategy and continue with selection on the basis of color after selection of the first letter, as predicted by the all-attributesare-equal theories (see our general introduction).

The conclusion that the situation for the all-attributesare-equal theories is not as desperate as Tsal and Lavie's (1988) research suggested is independent of the exact interpretation of Tsal and Lavie's results and the results of our Experiment 6. In the present study, we followed Tsal and Lavie's suggestion that these results might have been produced by fixation shifts to an array position prior to stimulus exposure. The fact that in the present study firm evidence for a switch from selection on the basis of color to selection on the basis of position was obtained only in the absence of fixation controls and under less favorable exposure conditions, that is, conditions in which identification performance can benefit from acuity enhancements as a result of appropriate eye fixations, clearly supports this suggestion. However, the fact that, under these conditions, fixation shifts might be helpful and the fact that, under these conditions, evidence is obtained for a switch from selection on the basis of color to selection on the basis of position, only suggest and certainly do not prove that subjects did, indeed, shift fixation prior to stimulus exposure.

In fact, for an explanation of Tsal and Lavie's results and the results obtained in our Experiment 6, the assumption that subjects shifted fixation is not really needed. There is an alternative explanation for the position-selection data which has, as a starting point, the asumption that subjects correctly fixated in the middle. ${ }^{2}$ The explanation starts by assuming that, in the absence of a fixation control task, subjects start out with distributed attention. The explanation continues by assuming that, upon stimulus presentation, subjects zoom in on one of the target locations or on a randomly selected position in the array and then process the colors and identities of the letters in that region of the array. Even when subjects focus a region of the array randomly, that region will nearly always contain a letter of the requested color (see our general introduction). When subjects name that letter first and additionally some other letters from that region, the results reported by Tsal and Lavie and found in our Experiment 6 will be obtained.

This explanation is new and certainly deserves further investigation; the fixation-shift versus proper-fixation issue requires eye-movement registration and cannot be decided on the basis of the data presented here. The important point, however, is that the outcome of such an investigation does not really change the theoretical conclusions that can be drawn from the present series of ex- periments. With this alternative explanation for Tsal and Lavie's (1988) results and the results obtained in our Experiment 6 , the important evidence remains that, in the present type of task, subjects prefer and tend to select on the basis of color, as predicted by the all-attributes-areequal theories; in our Experiment 5, with adequate exposure conditions and without a fixation-control task, the results obtained were nearly the same as those in our Experiments 1 and 2 with a fixation-control task (see also the Discussion of Experiment 5). It is only under the less favorable exposure conditions, such as those used in Experiment 6, that, in the absence of a fixation-control task, subjects prefer one or another region-selection strategy. (Our Experiment 3 showed that, with correct fixation, selection on the basis of position is difficult but not impossible.) So, for the present type of task, except under less favorable exposure conditions, selection on the basis of color seems to be the preferred or "most natural" strategy.

It will be clear, then, that the outcome of our investigations supports the all-attributes-are-equal theories. However, the results do not form a major threat to the position-special theories. The reason is that all contemporary major position-special theories contain one or another provision that allows them to account for selection on the basis of color (and other nonspatial attributes). In Treisman's $(1988,1990)$ feature-integration theory, for instance, relevant nonspatial attributes, such as color, can signal their positions in a master map of locations. Selective attention, operating in this master map, can then restrict its operations to the relevant locations in that master map (for related proposals, see, e.g., Cave \& Wolfe, 1990; van der Heijden, 1992, 1993, 1996; Wolfe, 1994, 1996; Wolfe et al., 1989). To account for the selection results presented in this study, this type of model can simply assume that (1) the instruction "name first a letter with color X," together with the presentation of the stimulus, has the result that all positions with color $\mathrm{X}$ are marked, in one way or another, in a position map, and (2) that selective attention then deals only with these marked positions (for details, see, e.g., Cave \& Wolfe, 1990; Treisman, 1988; van der Heijden, 1992).

With regard to the position-special versus all-attributesare-equal issue, then, Tsal and Lavie's (1988) ingenious paradigm appears to be an asymmetric paradigm. The paradigm and the results reported by Tsal and Lavie constituted a major threat for the all-attributes-are-equal theories and provided firm support for the position-special theories. The same paradigm and the results reported in the present study are compatible with the all-attributesare-equal theories but do not constitute a major blow for the contemporary, mature, position-special theories. To decide between the two groups of theories, alternative ingenious paradigms are needed (see, e.g., van der Heijden, 1993, for some suggestions).

One such paradigm is the partial-report task with color as the selection criterion. The most frequent type of error in these tasks is the near-location error; the incorrect responses tend to come from positions immediately adja- 
cent to the target (see, e.g., Fryklund, 1975; Snyder, 1972; van der Heijden, 1993). The explanation for these nearlocation errors is easy and straightforward in terms of the position-special theories and is difficult, but not really impossible, in terms of the all-attributes-are-equal theories. In fact, this observation follows directly from the position-special theories but requires additional, ad hoc, assumptions in the all-attributes-are-equal theories. This paradigm, however, needs much additional investigation (see van der Heijden, 1993, for further details).

The results of our series of experiments add to and extend the abundant evidence available that shows that attention can be directed toward elements of a particular color (see, e.g., Bundesen et al., 1984; Bundesen et al., 1985; Clark, 1969; Dick, 1969; Francolini \& Egeth, 1980; Fryklund, 1975; Humphreys, 1981; Kaptein et al., 1995; McClean, Broadbent, \& Broadbent, 1982; Nissen, 1985; Snyder, 1972; von Wright, 1968, 1970, 1972).

The present research adds to this evidence because the results show that subjects can select on the basis of color when forced by instruction to do so, as is the case with the first letter they were instructed to name in the Tsal and Lavie (1988) paradigm. In addition, the results of Experiment 4 show that, in response to a color cue, subjects can swiftly and flexibly direct their attention on a trialby-trial basis to an element with a relevant color (see also Kaptein et al., 1995).

The present research extends the evidence with regard to selection on the basis of color, because Experiments $1,2,4$, and 5 show that subjects even prefer to select on the basis of color when selection on the basis of color is no longer required by the instruction, as is the case with the free-report letters in the Tsal and Lavie (1988) paradigm. And Experiment 3 makes clear why subjects select these additional free-report letters on the basis of color. After the selection of a letter of a prespecified color, it is simply easier to continue to make selections on the basis of that color than to change to selection on the basis of position.

With regard to the study of selection in vision, Tsal and Lavie's (1988) paradigm still appears to be an important and promising one. The revelation of what selection strategy subjects prefer in the free report part can inform us about the relative difficulty of various selection schedules (see also Tsal \& Lavie, 1988).

\section{REFERENCES}

Broadbent, D. E. (1958). Perception and communication. London: Pergamon Press.

Broadbent, D. E. (1971). Decision and stress. London: Academic Press.

Bundesen, C. (1990). A theory of visual attention. Psychological Review, 97, 523-547.

BUNDESEN, C. (1991). Visual selection of features and objects: Is location special? A reinterpretation of Nissen's (1985) findings. Perception \& Psychophysics, 50, 87-89.

Bundesen, C., Pedersen, L. F., \& Larsen, A. (1984). Measuring efficiency of selection from briefly exposed visual displays: A model for partial report. Journal of Experimental Psychology: Human Perception \& Performance, 10, 329-339.
Bundesen, C., Shibuya, H., \& Larsen, A. (1985). Visual selection from multielement displays: A model for partial report. In M. I. Posner \& O. S. M. Marin (Eds.), Attention and performance $X I$ (pp. 631-649). Hillsdale, NJ: Erlbaum.

CAVE, K. R., \& WolfE, J. M. (1990). Modeling the role of parallel processing in visual search. Cognitive Psychology, 22, 225-271.

CLARK, S. E. (1969). Retrieval of color information from preperceptual memory. Journal of Experimental Psychology, 82, 263-266.

CoHeN, A., \& RAFAL, R. D. (1991). Attention and feature integration: Illusory conjunctions in a patient with a parietal lobe lesion. Psychological Science, 2, 106-110.

Dick, A. O. (1969). Relations between the sensory register and shortterm storage in tachistoscopic recognition. Journal of Experimental Psychology, 82, 279-284.

Egeth, H. E., Jonides, J., \& Wall, S. (1972). Parallel processing of multielement displays. Cognitive Psychology, 3, 674-698.

ERIKSEN, C. W. (1990). Attentional search of the visual field. In D. Brogan (Ed.), Visual search (pp. 3-19). London: Taylor \& Francis.

ERIKSEN, C. W., \& Hoffman, J. E. (1974). Selective attention: Noise suppression or signal enhancement? Bulletin of the Psychonomic Society, 4, 587-589.

ERiksen, C. W., \& Rohrbaugh, J. W. (1970). Some factors determining efficiency of selective attention. American Journal of Psychalogy, 83, 330-343.

Felleman, D. J., \& van Essen, D. C. (1991). Distributed hierarchical processing in the primate cerebral cortex. Cerebral Cortex, 1, 1-47.

Francolini, C. N., \& EGeTH, H. E. (1980). On the non-automaticity of "automatic" activation: Evidence of selective seeing. Perception \& Psychophysics, 27, 331-342.

FRYKLUND, I. (1975). Effects of cued-set spatial arrangement and targetbackground similarity in the partial-report paradigm. Perception \& Psychophysics, 17, 375-386.

HumphrEYs, G. W. (1981). Flexibility of attention between stimulus dimensions. Perception \& Psychophysics, 30, 291-302.

KaHNEMAn, D. (1973). Attention and effort. Englewood Cliffs, NJ: Prentice-Hall.

Kaptein, N. A., Theeuwes, J., \& van der Heijden, A. H. C. (1995). Search for a conjunctively defined target can be selectively limited to a color-defined subset of elements. Journal of Experimental Psychology: Human Perception \& Performance, 21, 1053-1069.

LA BERGE, D. (1983). Spatial extent of attention to letters in words. Journal of Experimental Psychology: Human Perception \& Performance, 9, 371-379.

LA Berge, D., \& Brown, V. (1989). Theory of attentional operations in shape identification. Psychological Review, 96, 101-124.

la Berge, D., Brown, V., Carter, M., Bash, D., \& Hartley, A. (1991). Reducing the effects of adjacent distractors by narrowing attention. Journal of Experimental Psychology: Human Perception \& Performance, 17, 65-76.

McClean, J. P., Broadbent, D. E., \& Broadbent, M. H. P. (1982). Combining attributes in rapid serial visual presentation tasks. Quarterly Journal of Experimental Psychology, 35A, 171-186.

NeISSER, U. (1967). Cognitive psychology. New York: AppletonCentury-Crofts.

Nissen, M. J. (1985). Accessing features and objects: Is location special? In M. I. Posner \& O. S. M. Marin (Eds.), Attention and performance XI (pp. 205-220). Hillsdale, NJ: Erlbaum.

Posner, M. I., SNYder, C. R. R., \& Davidson, B. J. (1980). Attention and the detection of signals. Journal of Experimental Psychology: General, 109, 160-174.

SNYDER, C. R. R. (1972). Selection, inspection and naming in visual search. Journal of Experimental Psychology, 92, 428-431.

Styles, E. A., \& Allport, D. A. (1986). Perceptual integration of identity, location and colour. Psychological Research, 48, 189-200.

THEEUWES, J. (1991). Categorization and identification of simultaneous targets. Acta Psychologica, 76, 73-86.

Treisman, A. M. (1988). Features and objects: The fourteenth Bartlett memorial lecture. Quarterly Journal of Experimental Psychology, 40A, 201-237.

Treisman, A. M. (1990). Variations on the theme of feature integration: Reply to Navon (1990). Psychological Review, 97, 460-463. 
Treisman, A. M., \& Gormican, S. (1988). Feature analysis in early vision: Evidence from search asymmetries. Psychological Review, 95, $15-48$

Treisman, A. M., \& Sato, S. (1990). Conjunction search revisited. Journal of Experimental Psychology: Human Perception \& Performance, 16, 459-478.

Tsal, Y., \& Lavie, N. (1988). Attending to color and shape: The special role of location in selective visual processing. Perception \& Psychophysics, 44, 15-21.

VAN DER HEIJDEN, A. H. C. (1992). Selective attention in vision. London and New York: Routledge.

van der Heijden, A. H. C. (1993). The role of position in object selection in vision. Psychological Research, 56, 44-58.

van der Heijden, A. H. C. (1996). Selective attention as a computational function. In A. F. Kramer, M. G. H. Coles, \& G. D. Logan (Eds.), Converging operations in the study of visual selective attention (pp. 459-482). Washington, DC: American Psychological Association.

VON WRIGHT, J, M. (1968). Selection in visual immediate memory. Quarterly Journal of Experimental Psychology, 20, 62-68.

VON WRIGHT, J. M. (1970). On selection in visual immediate memory. Acta Psychologica, 33, 280-292.

VON WRIGHT, J. M. (1972). On the problem of selection in iconic memory. Scandinavian Journal of Psychology, 13, 159-171.
WoLfE, J. M. (1994). Guided search 2.0: A revised model of visual search. Psychonomic Bulletin \& Review, 1, 202-238.

WOLFE, J. M. (1996). Extending guided search: Why guided search needs a preattentive "item map." In A. F. Kramer, M. G. H. Coles, \& G. D. Logan (Eds.), Converging operations in the study of visual selective attention (pp. 247-270). Washington, DC: American Psychological Association.

Wolfe, J. M., CAVE, K. R., \& Franzel, S. L. (1989). Guided search: An alternative to the feature integration model for visual search. Journal of Experimental Psychology: Human Perception \& Perfor. mance, 15, 419-433.

\section{NOTES}

1. The authors are grateful to Yehoshua Tsal, Tel Aviv University, for providing examples of the stimulus cards.

2. The authors are indebted to Kyle Cave and Yehoshua Tsal for providing this suggestion.

(Manuscript received December 4, 1995; revision accepted for publication February $15,1996$. 